\title{
WIDENING AUDIENCES - MAKING HERITAGE RECORDING DATA EASILY ACCESSIBLE VIA HTML APPLICATIONS
}

\author{
C. Boulanger ${ }^{\mathrm{a}}$, C. Ouimet ${ }^{\mathrm{a}}, \mathrm{S} . \mathrm{Kretz}^{\mathrm{a}}$, J. Gregg $^{\mathrm{a}}$ \\ ${ }^{a}$ Heritage Conservation Services, Public Services and Procurement Canada, 30 Rue Victoria, Gatineau, Quebec \\ (celine.boulanger, christian.ouimet, shawn kretz, john.gregg)@pwgsc.gc.ca
}

Commission II, WG II/8

KEY WORDS: Dissemination, high resolution photography, condition assessment, point cloud, HTML

\begin{abstract}
:
With the ever increasing size and complexity of heritage recording datasets, and consequently, the required expertise to manipulate and extract information for conservation projects from this data, the use of dissemination tools was researched and used to help bridge the gap between information gatherers and users in order to increase accessibility and utilization. This paper examines a variety of case studies where dissemination tools were utilized to make heritage recording data more easily accessible for a variety of users. The first example involves high resolution photography; the second explores methods of sharing large point cloud datasets; the third explores panoramic photography and dissemination via virtual tours; and the fourth, capitalizes on using panoramic images as a by-product of terrestrial laser scan data. All data was disseminated solely through the use of HTML outputs, ensuring that the end users did not require any specialized software and minimal to no training to visualize, manipulate and extract data from the assembled information. Collectively the project team felt that the simplicity of these outputs would increase the likelihood of their utilization by the various user groups. Based on the teams past experience, the requirement for specialized software greatly diminished the chances of broad use of the data by untrained individuals. By adopting a HTML platform, the difficulties wrought by software installation restrictions imposed on many organizations or limited by access to required hardware, could be greatly diminished. There is also a possibility for this type of data to be disseminated to the public for their interest using these tools; however, the presented examples show only how these methodologies were used in the understanding phase ${ }^{1}$ and execution stages by other professionals.
\end{abstract}

\section{INTRODUCTION}

Digital acquisition technologies such as record photography, panoramic photography, laser scanning and photogrammetry all form integral components of a comprehensive heritage record. As these technologies have developed, so too has the density of the resulting datasets which has an immediate impact on how they are managed and used resulting in the need to find and/or develop means to disseminate this data.

\section{HIGH RESOLUTION PHOTOGRAPHY}

\subsection{Case Study: Sinclair Centre}

The first case study showcases how a large number of high resolution photographs can be conglomerated and disseminated efficiently to various stakeholders and consultants involved in various projects at the Sinclair Centre in downtown Vancouver, British Columbia, Canada. This federally owned site is a monumental complex of four separate buildings covering an entire city block. It is both federally and municipally designated for its heritage values. The elevations of the building were documented with the intent of supporting a preliminary condition assessment. The location of the building on a prominent city block with electrified streetcar lines and mature trees on $50 \%$ of the perimeter streets and significant grade elevation changes on the property. This made it challenging to use a crane and/or manlift to access the upper portions of the facades and warranted alternative documentation solutions. The restrictive nature of the local municipal unmanned aerial system (UAS) permits further diminished access to the upper facades. Photography was the chosen method of documentation in this case and high resolution telephoto photographic images (Figure 1) were taken of all elevations using a Gigapan mount. This dataset comprised over 3,000 images, or 27 gigabytes of data. In order to make this large amount of data accessible to experts conducting the condition assessment, the photographs were stitched together to form mosaic panoramas of each elevation, varying from 1 to 2 gigapixels in resolution, and compiled to HTML outputs. As the data is parsed in the HTML output, it can be easily handled while preserving the high-resolution quality of the images when viewed zoomed-in at a high level of detail (Figure 3).

The high resolution images are efficiently delivered via the internet or intranet by creating a multi-resolution tiled image. The tiles ensure that the complete image does not need to be loaded, only the portion that is being visualised at each moment is actively rendered (Figure 2).

\footnotetext{
${ }^{1}$ Term coined in Standards and Guidelines (2010).
} 


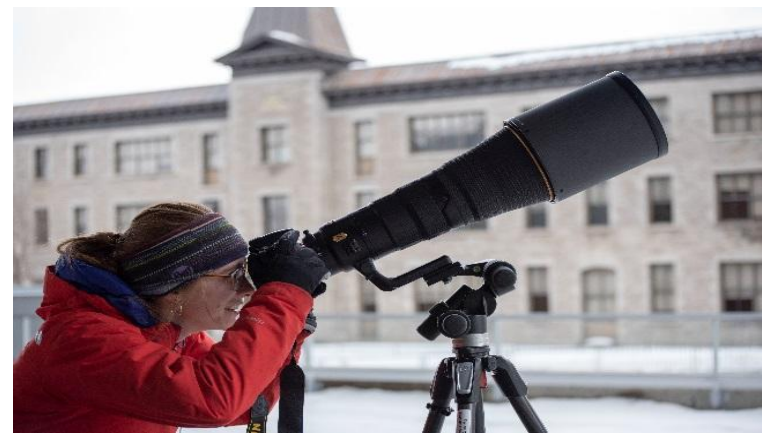

Figure 1: $600 \mathrm{~mm}$ lens used to capture high resolution imagery.

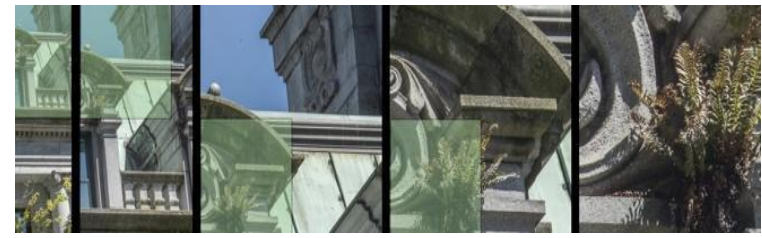

Figure 2: Multi-resolution tiled images for efficient dissemination.
The technique of stitching together images in a mosaic panorama can also be applied to disseminate traditional orthographic plans or elevations. Traditional architectural drawings are typically presented on a standard sheet size (A0, B1, etc...) at a specific scale. Depending on the chosen scale, the final output often does not take full advantage of the available resolution. Figures 4 and 5 display an orthographic image and deviation map of a historic aircraft hangar door sill, located in Ottawa, Ontario, Canada. These are examples of a traditional layout, and a tiled image viewable in a HTML format. The HTML format allowed the structural engineers analyzing the condition and deviation model to see the final output at greater detail than the PDF and paper versions could afford. Both the Sinclair Centre and aircraft hangar projects were produced using commercially available software, such as KRPano.

Through the use of the zoomable image it is straightforward to identify the location of a detail view, eliminating the need for separate image keys on elevations, and the HTML link can easily be shared. This tool also allows the possibility of adding information such as building condition notes which can be directly inputted onto the image with the use of clickable hotspots and the information further disseminated to subsequent users.

\subsection{Comparison to Traditional Methods}

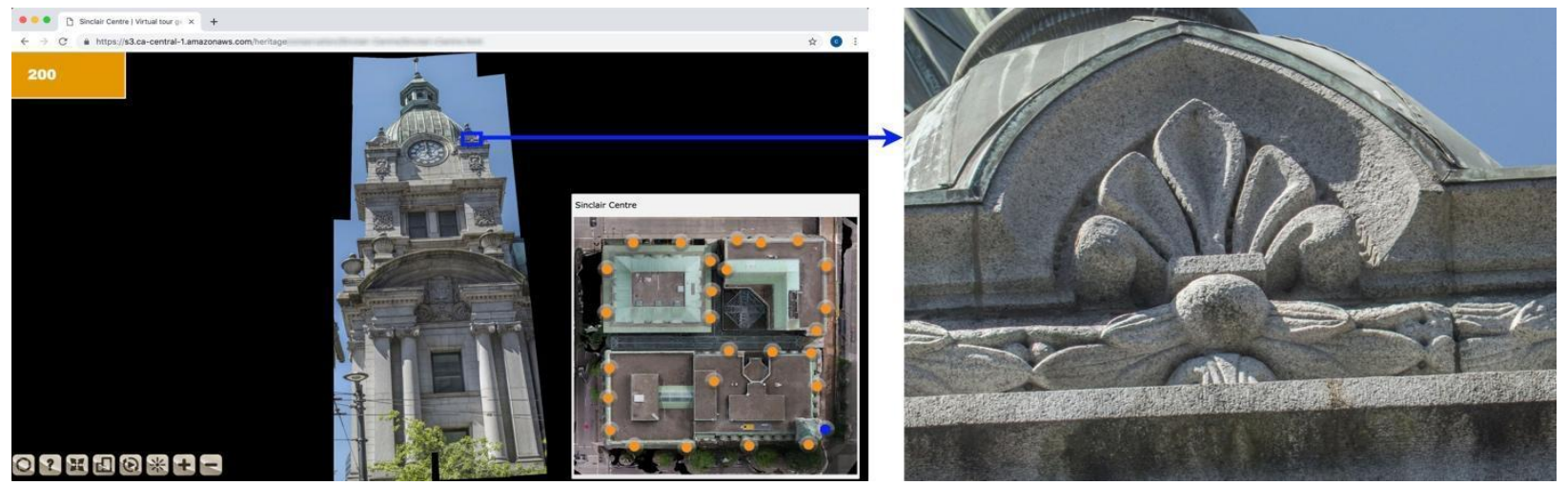

Figure 3: Key plan with clickable hotspots, 2 Gigapixel mosaic elevation, and zoomed in portion of image.

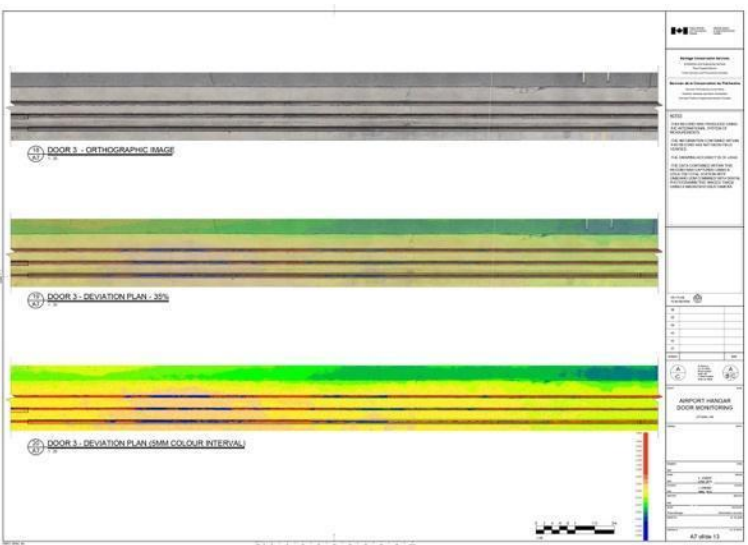

Figure 4: Traditional record drawing of a hangar door sill in plan at a specific scale.

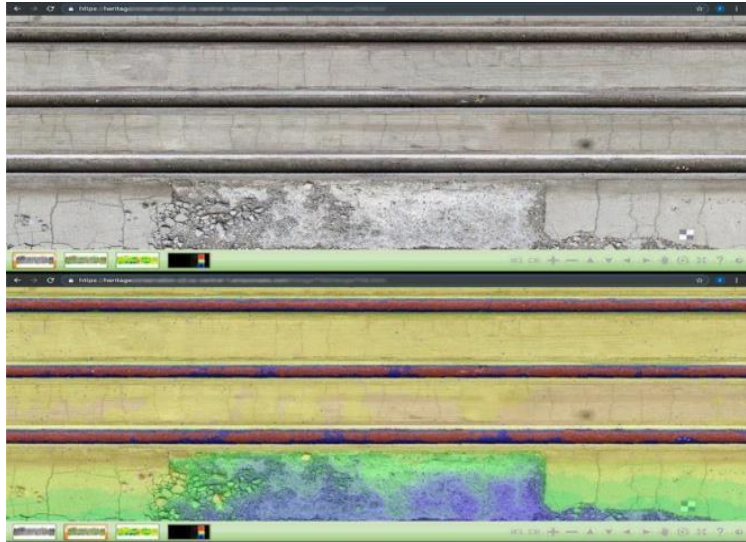

Figure 5: Interactive orthographic RGB image and related deviation map of the hangar door (from above). 


\section{DISSEMINATION OF POINT CLOUD DATASETS}

\subsection{Case Study: Dredge No. 4}

Dredge No. 4 National Historic Site of Canada "built in 1912 for the Canadian Klondike Mining Company, was the largest wooden hulled bucket-lined dredge in North America" (Parks Canada, 2018). This federally designated historic site is located 15.5 kilometres south of Dawson City, Yukon, Canada and is also part of the Klondike National Historic Sites of Canada (Figure 6). Aerial drone photogrammetry and terrestrial laser scanning were some of the tools employed to document the structure in a remote northern Canada site. Outputs consisted of two-dimensional line drawings and orthographic elevations. The laser scan data was disseminated via HTML outputs enabling users to better visualize and manipulate the point cloud data to suit the needs of their conservation work. (Figure 7). Open source software such as Potree, a point cloud renderer was used for this process. This renderer works similarly to the tiling process used for the high resolution photography. Linking additional pertinent heritage specific data, such as condition assessment notes, heritage character statements, etc. was also employed. This method of dissemination is particularly effective for this type of documentation output as it eliminates the need for specialized software typically needed, such as the point cloud viewer for example, Autodesk Recap. This facilitates file sharing due to the reduced size of the HTML link in comparison to the point cloud files, and diminishes the hardware requirements for the end user.

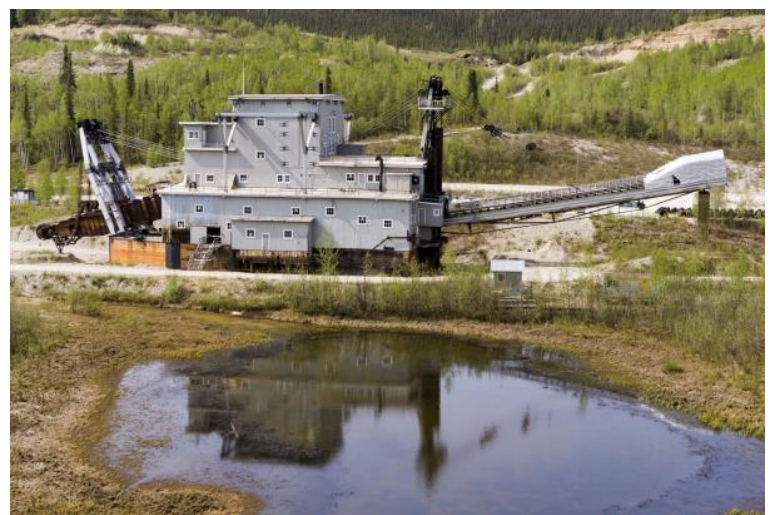

Figure 6: Dredge No. 4 National Historic Site of Canada.

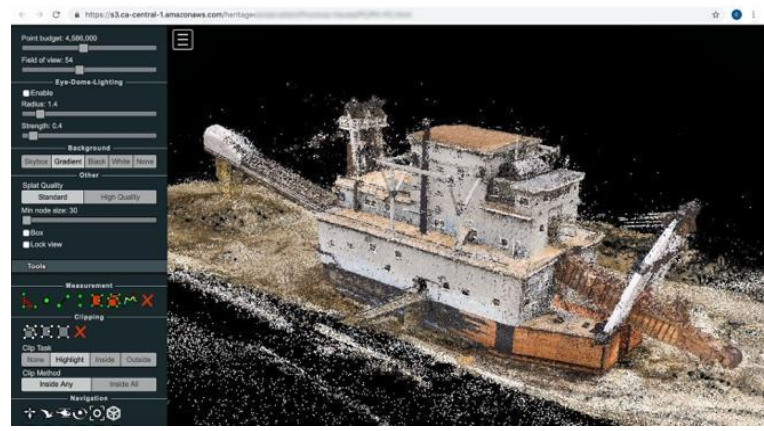

Figure 7: Point cloud project presented in an HTML format.

\section{PANORAMIC IMAGE DISSEMINATION}

\subsection{Panoramas Generated with the Use of Panoramic Cameras}

Panoramic images can be a useful tool to assist in the interactive visualization of a space or provide a wider angle of view, often at a much higher resolution than possible from a single frame image.

\subsubsection{Tools and Methods to Capture Panoramic Photography.}

There are a variety of tools or methods available on the market. The efficiency in which panoramas are captured and processed differs widely depending on the method employed. For example, a simple low-cost panoramic camera (Figure 8-a) can be used and inputs automatically stitched resulting in rapid capture and processing times. However, this method may not be suitable in areas with poor lighting conditions or if greater resolution is required. Another tool available for panoramic photography is a DSLR camera used with a panoramic tripod adapter (Figure 8-c). With this method, the resolution of the final output can be controlled by choosing lenses of different focal lengths. The ability to set up bracketed images in order to produce high dynamic range (HDR) photographs makes this method suitable for the capture of scenes with a wider range of lighting conditions. These images must be stitched and processed with independent software packages but produce a high quality product. In this case, the capture time is lengthier than with a low-cost panoramic camera and the post processing time can be substantial. Therefore, the use of this type of photography is often strategic and reserved for key spaces of high heritage significance where a high resolution is beneficial or there is value-added in capturing better information in areas with difficult lighting situations.

There are also a wide variety of mid-range panoramic cameras (Figure 8-b) on the market which offer balanced solutions relating to resolution, exposure and bracketing control. Although these cameras are typically limited by the overall quality of images that they produce; this is greatly offset by the time and inherent cost savings realized in the post processing stage as images are often automatically stitched and post-processed by proprietary software.

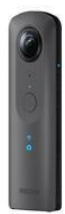

a

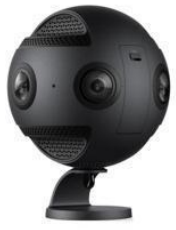

$\mathrm{b}$

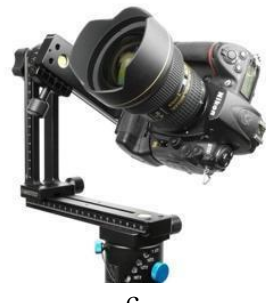

c
Figure 8: Various panoramic capturing options.

The use of mid-range cameras offering automated workflows allows for the complete capture of spaces in an efficient manner. 
4.1.2 Use of Panoramic Photography at Dredge No. 4: A mid-range panoramic camera was used at the site of Dredge No. 4. In this case, the entire structure was captured with nearly 350 panoramic images in a period of three days, averaging 3-4 minutes per position with setup and acquisition. This dataset, disseminated via an HTML portal, provides an effective and accessible visual tool complementary to metric information, such as laser scanning and photogrammetry, and assists greatly with the production of drawings or BIM models. This graphic information can also be used: in meetings relating to the cultural resource; by consultants or contractors submitting bids on potential conservation projects; and easily be disseminated to a wider audience such as the general public. This is especially useful for sites such as Dredge No. 4, which is considered remote and geographically difficult to access. It also serves as a value-added deliverable forming part of a posterity record.

\subsection{Panoramic Images Generated From Laser Scanning} The intensity values from laser scan point cloud data can be used in situations where panoramic images are not taken due to extremely difficult lighting conditions.

\subsubsection{Case Study: Centre Block of Parliament}

The Centre Block of Parliament in Ottawa, Ontario, Canada, has been extensively documented over the past several years in order to have adequate documentation records in advance of the planned rehabilitation project. Select spaces of the building were recorded using photography and photogrammetry. Furthermore, the building was fully documented using terrestrial laser scanning, including all public, office, basement and attic spaces, as well as the exterior of the building. This project consisted of well over 1000 individual scan positions. One of the by-products of terrestrial laser scanning was panoramic images generated from the intensity values of the scanner, or from the panoramic RGB images captured by the scanner.

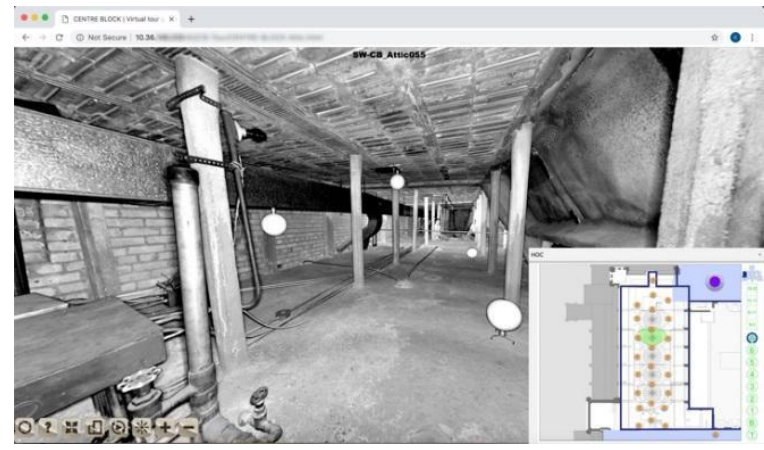

Figure 9: Lidar intensity value panoramic image (with key plan) of an attic space with limited to no lighting, showing visual information about the structure.

The intensity value panoramic images proved to be an excellent method to visualize the geometry of the structure in extremely dark areas, such as the attic or interstitial spaces behind the visible finishes. These are also the areas that generally yield the most valuable information regarding the structural composition of the building, such as the wall materials and exposed structural elements (Figure 9).

All scan positions were keyed to floor plans, and transferred to an HTML tour platform in order to disseminate the information (Figure 10). This information allows the endusers to quickly view any part of the building to confirm asfound site conditions, or spatial configurations. In a meeting environment this tool can help quickly answer questions and provide visual support to ensure that all team members have an understanding of the space, thus reducing chances of miscommunication and/or errors.

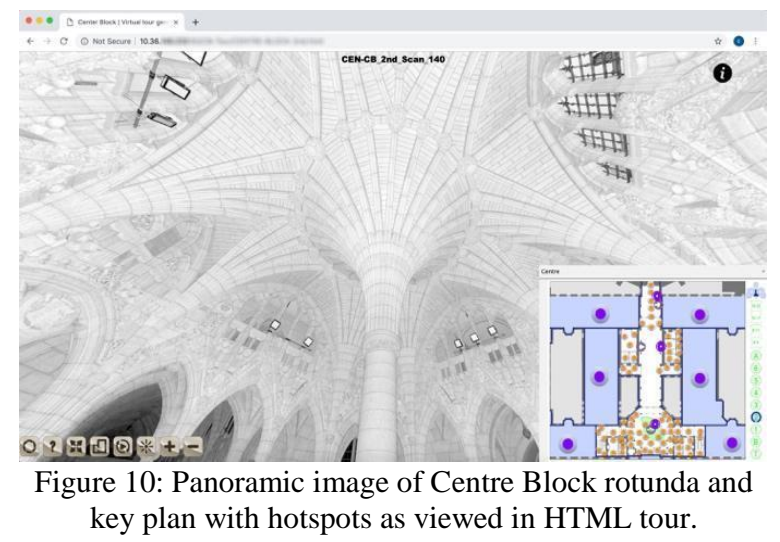

\subsection{Advantages of Disseminating Panoramas via HTML Outputs}

In comparison to traditional methods, dissemination via HTML outputs are advantageous for disseminating panoramas. Traditional methods include projecting panoramas onto a flat surface and keying panoramas on plans. These can be shared in printed or digital form. Projecting a sphere, or 360 degree view, onto a flat surface results in distortion to the image and "a panorama-maker (like a map-maker) has to determine what are the features that are more important to preserve in a panorama, and choose the projection accordingly" (German, 2007). These distortions can sometimes be difficult to interpret by the end user. This is resolved when the panoramas are disseminated via an HTML output as they can be mapped for a virtual tour. This creates an immersive experience for the end-users and enables them to pan and zoom around the panorama as if they are at the center of the space. In this type of output the distortion typically found in panoramas projected onto a flat surface is minimised.

Where traditional methods of dissemination can be cumbersome to navigate and inconvenient to share, dissemination via HTML platforms is intuitive to navigate and easily shareable. Traditional methods require users to refer back and forth from key plans to panoramas. If files are shared in printed format the resolution of the panos is limited to the size at which they are plotted. Sharing files digitally provides users with the full resolution of the panos but becomes difficult to share in substantial datasets due to their large file size. The HTML outputs in comparison are very intuitive to navigate as each panorama location can be a clickable hotspot on a plan which becomes highlighted when 
the users select a particular panorama. Navigational hotspots can even be added to allow users to jump directly to the next location from within their field of view. Overall, this immersive experience helps the end-uses gain a better understanding of the space, enables them to benefit from the full resolution of the panoramas and facilitates sharing the project to other user groups.

\section{CONCLUSION}

As the technology used to capture and compile heritage documentation evolves, the resulting datasets are ever increasing in size and overall complexity. Concurrently, dissemination technology is also changing as is the potential use of the data; in many instances these are not or have not been traditional uses of heritage records. The case studies discussed provide a selection of tools used to visualize, manipulate and extract data from the assembled information making these records widely available, and easily used by a variety of users without specialized software and minimal to no training. Advancements in both documentation and dissemination technology ultimately help improve the accuracy of information; communication, understanding and appreciation of the historic place; and, provides access to a greater audience. By making heritage recording datasets more accessible, collectively it will help contribute in the conservation of heritage places. In most instances using the tools and technology presented in the case studies of this paper, the projects have: experienced reduced costs; benefitted their respected project schedules by providing clarity to the greater project teams; and, have ultimately assisted the conservation process.

\section{REFERENCES}

Parks Canada, 2010. Standards and Guidelines for the Conservation of Historic Places, Second Edition. Canada. https://www.historicplaces.ca/en/pages/standardsnormes.aspx

Parks Canada, 2018. Dredge No. 4 National Historic Site. https://www.pc.gc.ca/en/lhn-nhs/yt/klondike/culture/lhnnhs_dn4

German et al., 2007. New methods to project panoramas for practical and aesthetic purposes, Computational Aesthetics in Graphics, Visualization and Imaging. The Eurographics Association. 